\title{
Enfoque del manejo de cuerpos extraños colorrectales: revisión de la literatura
}

\section{Literature Review of Management of Colorectal Foreign Bodies}

Carlos E. Martínez, ${ }^{1}$ Lina Mateus, ${ }^{1}$ Heinz Ibáñez, ${ }^{1}$ Nairo Senejoa, ${ }^{1}$ Anwar Medellín, ${ }^{2}$ Alexander Obando, ${ }^{2}$ Hernán Cardona, ${ }^{3}$

Jaime A. Montoya. ${ }^{4}$

Coloproctólogo del Hospital Militar Central. Bogotá D. C., Colombia.

2 Fellow de coloproctología de la Universidad Militar Nueva Granada. Bogotá D. C., Colombia.

3 Residente de cirugía general, Universidad Nacional de Colombia. Bogotá D. C., Colombia.

${ }_{4}^{4}$ Fellow de cirugía gastrointestinal y endoscopia digestiva del Instituto Nacional de Cancerología. Bogotá D. C., Colombia.

Correspondencia: Jaime A. Montoya. Correo: jaimemontoya6@hotmail.com.

Fecha recibido: $\quad 08-07-17$

Fecha aceptado: 22-01-18

\begin{abstract}
Resumen
La presentación de pacientes con cuerpos extraños a nivel rectal no es poco frecuente; sin embargo, hay pocas estadísticas sobre la epidemiología de esta entidad. La mayor parte de la literatura publicada hace mención a reporte de casos o series de casos. Los cuerpos extraños rectales son insertados de forma intencional o no intencional, y constituyen un reto diagnóstico y terapéutico para el médico; por lo que el médico a cargo requiere de la habilidad y conocimiento para extraer objetos de diferentes formas y tamaños. Los cuerpos extraños rectales son generalmente observados en la población adulta; en relación con la estimulación erótica 0 asalto sexual. Adicionalmente, algunos cuerpos extraños pueden ser ingeridos ocasionalmente, pasando a través del tracto gastrointestinal y alojándose en el recto, aunque esta situación es la menos común; en especial en pacientes con enfermedades mentales, trastornos visuales, alcohólicos o usuarios de prótesis dentales. La finalidad de esta revisión es establecer unas pautas de manejo y dar a conocer un algoritmo para el enfoque de los cuerpos extraños colorrectales.
\end{abstract}

\section{Palabras clave}

Enfermedades del recto, colon, cuerpo extraño.

\section{Abstract}

Patients with foreign bodies in their rectums are not uncommon, but statistics on the epidemiology of this entity are scarce. Most of the literature published consists of case reports or case series. Foreign rectal bodies, whether inserted intentionally or unintentionally, constitute a diagnostic and therapeutic challenge for physicians who need skill and knowledge to extract objects of different shapes and sizes.

Foreign rectal bodies are generally observed in the adult population in relation to erotic stimulation or sexual assault. Occasionally, foreign bodies are ingested and pass through the gastrointestinal tract to lodge in the rectum. Although this situation is the least common, it does occur, especially in patients with mental illnesses, visual disorders, or alcoholism and among users of dental prostheses. The purpose of this review is to establish management guidelines and to present an algorithm for the approach to colorectal foreign bodies.

\section{Keywords}

Diseases of the rectum, colon, foreign body. 


\section{LITERATURA E HISTORIA DE CUERPO EXTRAÑO RECTAL}

Citando un artículo de Haft y Benjamin (1) sobre los aspectos psicosociales de los cuerpos extraños rectales, los casos más antiguos datan de la Antigua Grecia o el Antiguo Egipto. El caso más antiguo reportado en una revista médica fue en el año 1919 (Smiley). Algunos artículos en libros médicos de proctología dan razón de la literatura de los cuerpos extraños (Gant, 1902) y tratados quirúrgicos (Poulet, 1881). El número de artículos que describen los cuerpos extraños se han incrementado en el transcurso del tiempo, de menos de 30 artículos publicados en la literatura antes de 1950 a 66 artículos de 2000 a 2015. La incidencia actual de los cuerpos extraños es desconocida. Tradicionalmente, se ha descrito una mayor incidencia en el sexo masculino, pero no hay relación de la orientación sexual con los cuerpos extraños rectales (a pesar de la información poco crítica y arbitraria desde la década de 1970 hasta la de 1990 en los que se asociaba con la población homosexual) (2). El diagnóstico más tardío documentado en la literatura fue de 5 años después de la inserción del cuerpo extraño (3).

\section{EPIDEMIOLOGÍA Y ETIOLOGÍA}

Hay una clara preponderancia del sexo masculino, con una relación que puede llegar a ser 28:1 y de predominio en el grupo etario entre los 20 y 40 años (4). La mayor parte de la retención de cuerpos extraños a nivel rectal tiene la finalidad de estimulación erótica y gratificación, un valor que puede alcanzar hasta un $75 \%$ y un $12,5 \%$ en relación con el asalto sexual. Hay algunos casos aislados reportados de retención de cuerpos extraños a nivel rectal en pacientes mayores $(60 \mathrm{a}$ 80 años) utilizados para el autotratamiento de la impactación fecal o masaje prostático. Otros, en el contexto de pacientes institucionalizados como prisioneros o pacientes psiquiátricos, pueden alojar elementos (cuchillos, drogas ilícitas o armas) con la finalidad de lesionar a sus cuidadores, guardas, pacientes o prisioneros (5). Los cuerpos extraños en niños son poco comunes y usualmente se relacionan con abuso sexual, por lo que se debe investigar. Casi cualquier objeto puede ser encontrado: desde juguetes sexuales, botellas, bombillos, elementos metálicos o de madera, frutas o vegetales, baterías, aerosoles, tapas u otros.

En un estudio retrospectivo realizado en Japón, desde 2007 a 2010 que incluía a 648 pacientes (de 431 hospitales), se encontró que 15 pacientes tuvieron más de un ingreso hospitalario por dicha causa $(2,3 \%)$, con un máximo de 4 readmisiones. Así mismo, el número de pacientes masculinos fue de $526(81,1 \%)$, con una relación de hombre-mujer de 4,3 a 1 (pacientes masculinos en un rango de edad de 60 a 69 y femeninos de 80 a 89 años). Existió una asociación de patología mental mayor en mujeres respecto a hombres ( $8,2 \%$ frente a $2,7 \%$ en hombres).

La mortalidad global fue de $1,2 \%$. De los 44 pacientes con perforación y peritonitis, uno de los casos fue iatrogénico. La proporción de muerte, perforación/peritonitis y sepsis para el grupo femenino fue de $4,8 \%, 12,8 \%$ y $4 \%$, respectivamente. Los pacientes masculinos fueron más proclives a ser llevados a la extracción de cuerpo extraño vía transanal que la vía transabdominal; alrededor del $73 \%$ de los pacientes recibieron anestesia general o regional (6).

Un análisis de regresión logística multivariable identificó que los pacientes femeninos tienen una posibilidad 2 veces mayor de tener peores desenlaces que los hombres (abscesos anales o rectales, perforación, sepsis o peritonitis). La perforación por ingesta de cuerpos extraños puede llegar a ser del $25 \%$ en el recto o el ano (7).

Así mismo, hay una relación poco clara entre los cuerpos extraños a nivel rectal con la infección por virus de la inmunodeficiencia humana (VIH). En Japón, la prevalencia de la infección por VIH en la población general es del 0,018\%, pero en el estudio se encontró una prevalencia de 1,1\%, lo que sería de importancia para realizar un examen serológico en este grupo poblacional, teniendo en cuenta que normalmente solo se realiza en el 10\% de esta subpoblación (6).

\section{CLASIFICACIÓN Y TIPOS}

Los cuerpos extraños se pueden clasificar de diferentes maneras. Según su ubicación, se pueden encontrar en el tracto gastrointestinal superior, definido como el intestino delgado, donde generalmente se ingieren y se impactan en las zonas anatómicamente más estrechas; cuando estos cuerpos logran pasar al colon, se clasifican como del tracto gastrointestinal inferior, que pueden ser retenidos tanto en el colon como en el recto. Para los cuerpos que se introducen a través del recto se deben considerar varias características anatómicas como los esfínteres anales interno y externo, que se contraen tónicamente y pueden estar edematizados después de la inserción; también la curvatura natural en la unión recto-sigmoidea, que puede causar dificultad para el paso espontáneo y la eliminación (8). Teniendo en cuenta lo anterior, para fines prácticos de manejo, se propone valorar la ubicación del cuerpo extraño por encima o por debajo de promontorio.

Los cuerpos extraños también se describen como voluntarios frente a involuntarios y sexuales frente a no sexuales (Tabla 1) (9). Lo anterior cobra importancia porque da una orientación acerca del tipo de trauma que puede tener el paciente, y esto se relaciona íntimamente con el objeto, que debido a la gran variedad de tamaños y formas, amplía el espectro de las lesiones y las técnicas para su extracción (10). En la literatura aparecen reportados vibradores, dil- 
dos (consoladores), pepinos, manzanas, bombillos, frascos de vidrio y plásticos, huesos, entre otros.

Tabla 1. Clasificación de cuerpos extraños

\begin{tabular}{lll}
\hline & Voluntarios & \multicolumn{1}{c}{ Involuntarios } \\
\hline Sexuales & $\begin{array}{l}\text { Vibradores, dildos y otra } \\
\text { variedad de objetos }\end{array}$ & Violación o asalto \\
No sexuales & $\begin{array}{l}\text { Embalaje en el cuerpo } \\
\text { de drogas ilícitas }\end{array}$ & $\begin{array}{l}\text { Enfermos mentales, niños } \\
\text { o ingesta de huesos, } \\
\text { plásticos, entre otros }\end{array}$ \\
\hline
\end{tabular}

Según las características del cuerpo extraño, puede usarse la clasificación de fácil agarre, dentro de los que se encuentran elementos cortopunzantes pequeños, huesos, espinas y fálicos como los consoladores, vibradores, velas, vegetales y frutas (Figura 1). Los de difícil agarre corresponden a envases, botellas, tarros, frascos, esferas, bombillos, entre otros (Figura 2).

También es importante tener en cuenta la clasificación de trauma de recto (rectal organ injury scale) de la Asociación Americana para la Cirugía del Trauma (AAST) para definir el manejo.

\section{CUADRO CLÍNICO}

El diagnóstico puede ser difícil por vergüenza de los pacientes, que conlleva a que acudan a los servicios de urgencias de manera tardía $(1,11,12)$. Es importante obtener información precisa sobre el elemento introducido, aunque difícilmente el paciente la suministrará en los episodios de autointroducción.

En la historia, el síntoma cardinal es el dolor anal o constipación. Otros síntomas descritos son el sangrado, incapacidad para la micción, dolor abdominal en cuadrantes inferiores, incontinencia anal, diarrea, múltiples intentos fallidos de extraer el cuerpo extraño, sepsis en algunos pacientes (hipotensión, taquicardia e irritación peritoneal) en caso de perforación, o con colapso cardiovascular en pacientes que son utilizados para tráfico de sustancias psicoactivas y que presenten rupturas de las mismas; en este tipo de paciente, la información también será difícil de obtener $(12,13)$.

La duración de la retención del objeto en la región anorrectal se relaciona directamente con el riesgo de ruptura y de lesión de la mucosa.

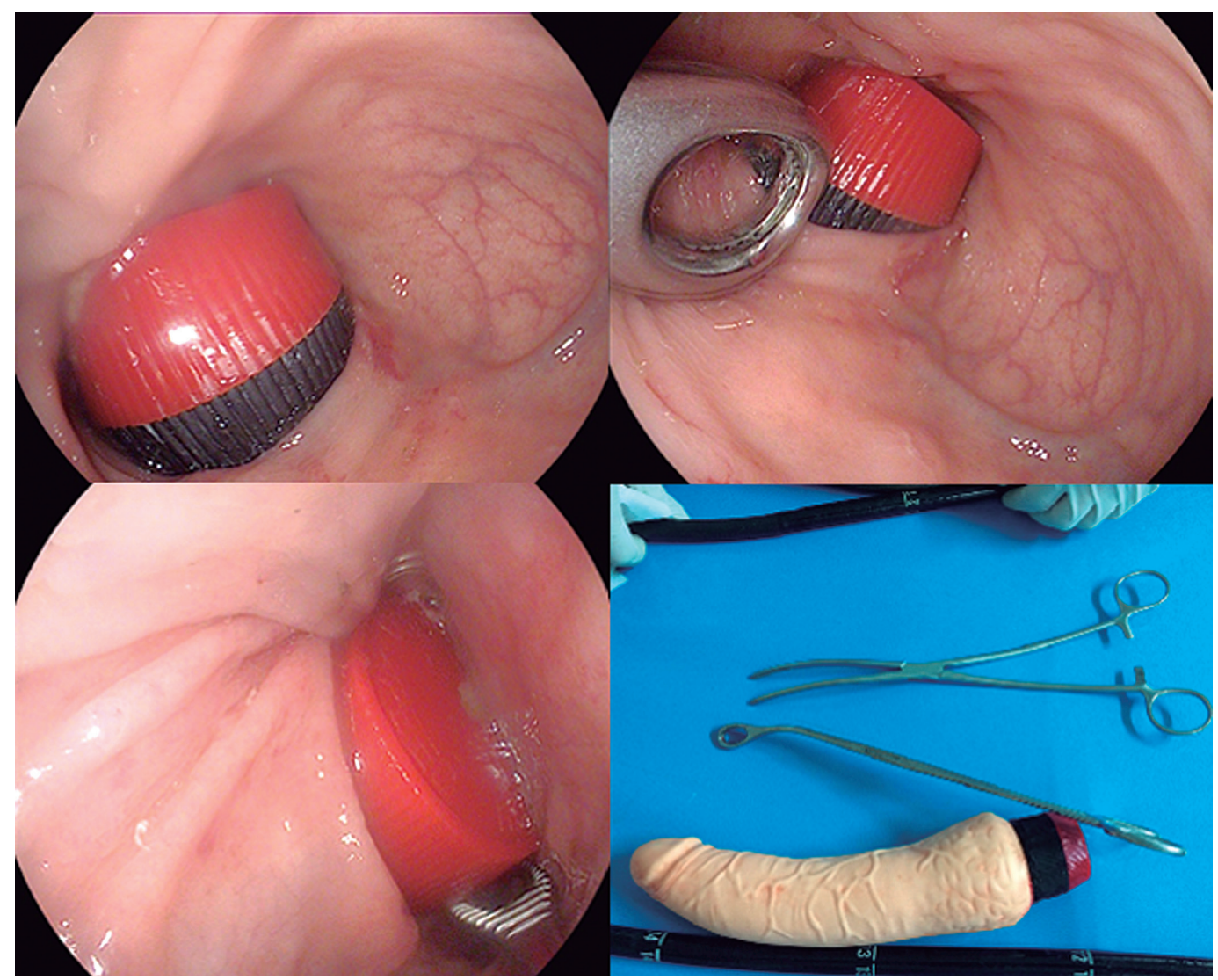

Figura 1. Cuerpo extraño de fácil agarre. 


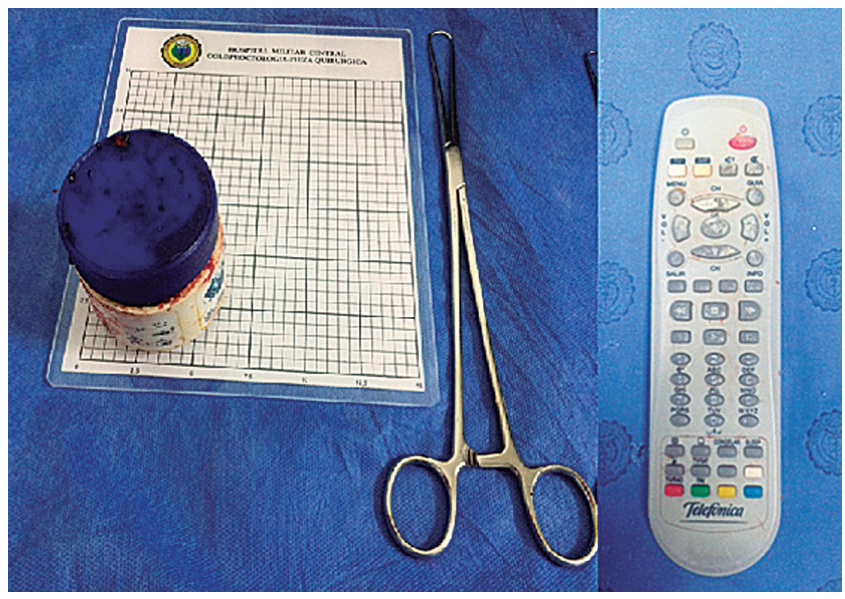

Figura 2. Cuerpo extraño de difícil agarre.

\section{EXAMEN FÍSICO}

La privacidad del paciente debe mantenerse, evitar de cualquier modo realizar comentarios cómicos o discriminatorios, y proteger la intimidad del paciente por parte del personal asistencial curioso que no tenga relación con la atención (10).

El objetivo principal de la evaluación inicial es identificar el tipo, número, tamaño, forma y localización del cuerpo extraño (10). La importancia de una descripción detallada del objeto es fundamental para poder establecer la mejor opción de manejo; sin embargo, se requiere un adecuado acercamiento por parte del examinador, para lograr la descripción del mismo. El personal médico debe ser respetuoso y gentil para lograr establecer una relación adecuada basada en la confianza, además de no realizar juicios; es fundamental dar una explicación clara con el fin de lograr una comunicación acertada y que el paciente esté convencido de la conveniencia de dar información relevante acerca del mecanismo y tipo de objeto (14).

Debe realizarse un examen cuidadoso y detallado evaluando el abdomen desde la inspección, pasando por la auscultación, en la cual se puede documentar peristaltismo aumentado, disminuido o ausente. Uno de los fines de esta evaluación es descartar la presencia de signos de irritación peritoneal. En algunas ocasiones, se pueden palpar los cuerpos extraños y en el área perineal deben describirse claramente hallazgos como la presencia de laceraciones, hematomas, perforaciones, sangrado, presencia o no de malos olores, descarga de secreción mucoide o sanguinolenta. En caso de presentarse salida de líquido mucoide de aspecto chocolate fétido, sugiere la presencia de necrosis, perforación o sepsis (15).

$\mathrm{Al}$ realizar el tacto rectal, debe tenerse cuidado al introducir el dedo ya que algunos objetos pueden ser punzantes o cortantes, lo que genera un riesgo de accidente biológico por parte del personal médico. En este se debe detallar el estado del tono del esfínter anal; la evaluación debe realizarse antes y después de la extracción del cuerpo extraño. El espasmo involuntario puede ocurrir durante el intento de extracción. Esta información es sumamente relevante y debe quedar claramente consignada en la historia clínica.

En los pacientes sin lesión del esfínter, el tono se debe encontrar aumentado como resultado del espasmo muscular secundario al objeto. El cuerpo extraño puede ser directamente palpado; en caso de no palpar el objeto, hallazgos como salida de material hemático o pérdida del tono del esfínter hacen sospechar la presencia de un cuerpo extraño $(9,16)$. Si se presenta una perforación por encima del recto superior, el paciente tiene signos clínicos de peritonitis.

Cuando los hallazgos en el tacto rectal son negativos o se requiere mejorar la visualización, se puede usar la anoscopia; su limitante se encuentra en el diámetro del mismo, pero permite identificar la naturaleza y posición exacta del objeto.

En caso de cualquier duda con respecto a la integridad de la mucosa anorrectal, se debe realizar una evaluación dirigida.

\section{ESTUDIOS DIAGNÓSTICOS}

Las radiografías $(\mathrm{Rx})$ de abdomen son los exámenes de primera mano para hacer el abordaje inicial; estas permiten localizar el objeto, estimar el tamaño y determinar la cantidad. Se requiere usar una proyección anteroposterior y otra lateral para dar una localización más exacta (Figuras 3 y 4). Los objetos como el metal o grava son más densos que los tejidos que los rodean y permiten una mejor visualización en las Rx. Los materiales orgánicos que poseen una densidad similar a los tejidos humanos pueden no ser observados en las diferentes proyecciones (9). La no observación de un cuerpo extraño en la $\mathrm{Rx}$ no descarta la presencia del mismo.

La Rx de tórax de pie debe ordenarse en todo paciente en quien se sospecha una perforación intraperitoneal.

En caso de no visualizarse el objeto en las $\mathrm{Rx}$, se puede emplear una colonoscopia, tomografía axial computarizada (TAC) de abdomen y pelvis, y estudios con medio de contraste hidrosoluble, teniendo la precaución de no aumentar demasiado la presión hidrostática que podría perforar la pared.

El cuadro hemático y los parámetros inflamatorios como la proteína C-reactiva (PCR) y la velocidad de sedimentación globular no son exámenes rutinarios iniciales, pueden ser empleados en los casos de sospecha de perforación. Se ha descrito la utilización de gases arteriales para determinar acidosis en pacientes sépticos. En caso de sospecha de intoxicación, se debe realizar un rastreo toxicológico (10).

La ultrasonografía endoscópica se ha descrito como método diagnóstico en el caso de cuerpos extraños impactados que 


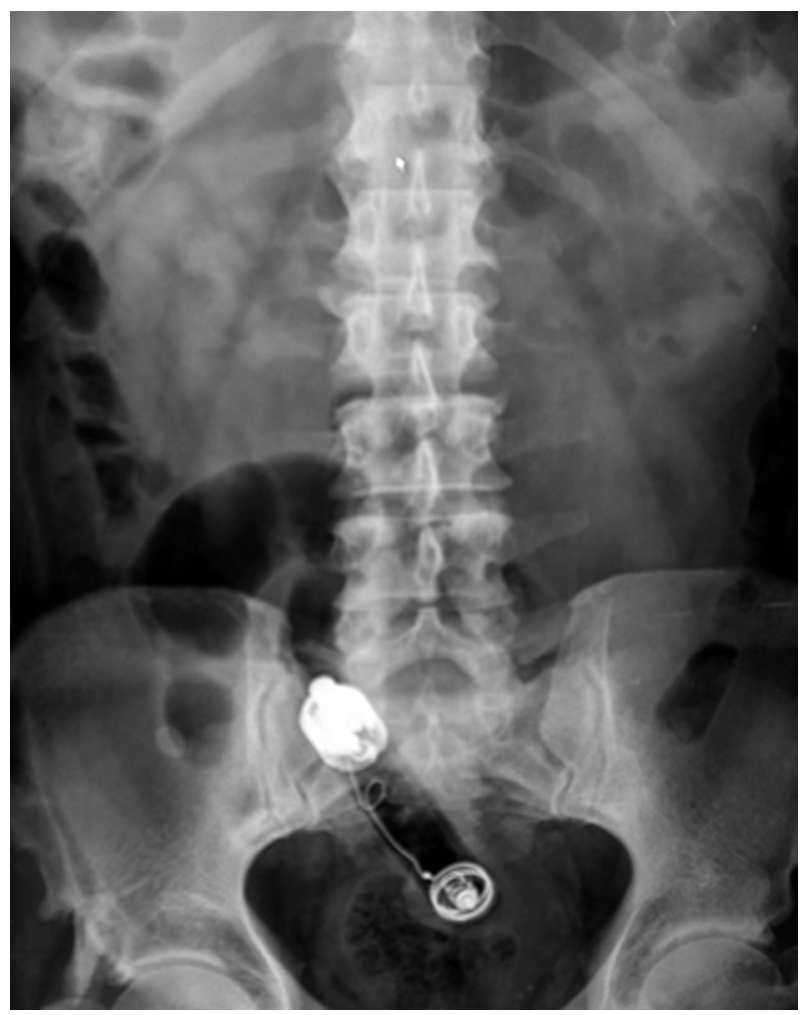

Figura 3. Rx de abdomen anteroposterior.

generan lesiones similares a las subepiteliales; documentando granulomas y el cuerpo extraño en el interior (17).

La tomografía contrastada de abdomen y pelvis está indicada cuando se sospecha perforación por debajo de la reflexión peritoneal. En caso de sospecha de perforación extraperitoneal de recto, los hallazgos que pueden observarse son adelgazamiento de la pared rectal, aire mesorrectal, líquido perirrectal o estriación de la grasa mesorrectal.

La endoscopia provee una excelente visualización de los segmentos evaluados y permite realizar el diagnóstico y extracción de los mismos. Requiere insuflación que logra bloquear el fenómeno de válvula producido cuando el objeto se adosa a la mucosa. Es necesario realizar rectosigmoidoscopia flexible luego de la extracción para descartar lesiones del colon o recto, y que el paciente no se haya introducido más objetos $(8,10)$.

\section{TÉCNICAS}

En pacientes con abdomen agudo quirúrgico en los cuales se sospeche perforación están indicados la hidratación parenteral vigorosa, inicio de antibióticos de amplio espectro, sonda vesical y sonda nasogástrica (SNG) descompresiva para ser llevados a laparotomía exploratoria.

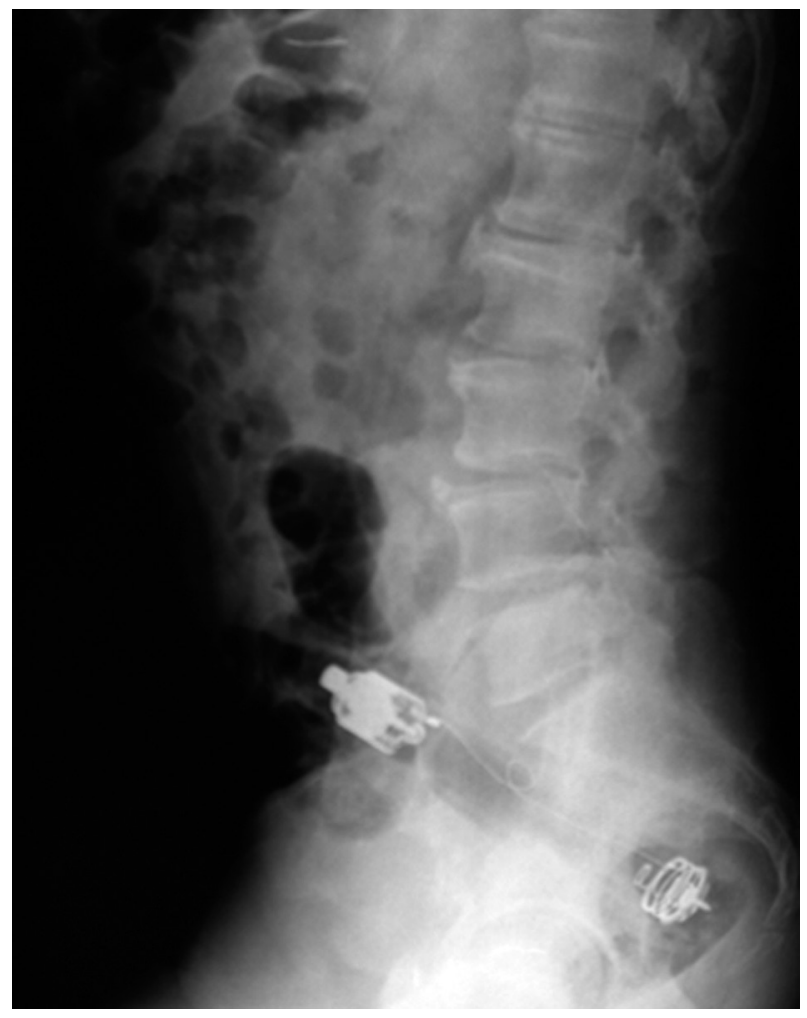

Figura 4. Rx de abdomen lateral.

Una de las medidas importantes a tener en cuenta para realizar la extracción a través de este método es la relajación. En caso de tener un paciente colaborador y tranquilo, puede intentarse el procedimiento sin anestesia; de lo contrario, se puede realizar con anestesia local que produce el bloqueo de los nervios perianales, sedación consciente, anestesia raquídea y, en última instancia, anestesia general, que busca la relajación, eliminación del espasmo del esfínter y mejoramiento de la visualización y exposición.

Para la anestesia local se puede emplear lidocaína con epinefrina al $1 \%$ o bupivacaína al $0,5 \%$ con epinefrina o una mezcla de ambas en relación $1: 1$. Se realiza un bloqueo inicialmente superficial, luego interesfintérico circunferencial y, ulteriormente, se bloquea el nervio pudendo. Este último se logra infiltrando los tejidos profundos en la cara posterolateral bilateral a $1 \mathrm{~cm}$ medial de las tuberosidades isquiáticas. Después se debe realizar dilatación digital de manera gentil.

No se recomienda retirar a ciegas objetos que posean alto riesgo de lesionar el recto por los bordes afilados, ruptura o fragmentación; por ejemplo, bombillos, vasos de cristal, entre otros.

Se plantea que la extracción en la misma cama de evaluación tiene una tasa de éxito entre el 60\% al 75\%, aunque se ha 
descrito una alta tasa de fallos con la extracción transanal además de empujar los objetos hacia la región rectosigmoidea.

El procedimiento comienza en el servicio de emergencia con la evaluación inicial en la que los objetos pequeños se pueden enganchar; en pacientes que no tengan irritación peritoneal, el dedo deber lubricarse adecuadamente con lidocaína jalea y realizar presión abdominal con el fin de lograr movilizar distalmente el objeto. Debe evitarse de cualquier modo empujar el objeto hacia el recto proximal.

Se han planteado factores predictores de fallo de extracción dentro de los cuales están: objetos de más de $10 \mathrm{~cm}$ de largo, objetos duros o afilados, aquellos que migren o que lleven más de 2 días de retención (12).

Para los objetos de fácil agarre se realiza anoscopia: retirarlos con pinza de Kelly o Rochester:

- Anestesia: sin anestesia o anestesia local.

- Posición: navaja sevillana.

- Evitar laceraciones de la mucosa.

En aquellos de difícil agarre se realiza:

- Anestesia: local o regional.

- Equipo para visualizar: anoscopio, separador anal Pratt, rectoscopio rígido, colonoscopia.

- Equipo de agarre: pinza de Foerster o pinza de cuerpo extraño.

- Posición: litotomía, lateral.

- Maniobra: presión abdominal en hipogastrio.

Debe tenerse cuidado con los pacientes no colaboradores o ansiosos en quienes el dolor se asocia con espasmo del esfínter anal; esto limita los intentos para retirarlo en los servicios de emergencias.

En caso de que la extracción transanal falle, hay 2 aproximaciones mínimamente invasivas antes de la exploración abdominal: la sigmoidoscopia flexible y el TAMIS (cirugía transanal mínimamente invasiva). Esta última tiene 3 ventajas: muchos cirujanos pueden emplearla por estar habituados al uso de la laparoscopia, la imagen magnificada del objeto permite además definir el estado de la mucosa y no se requieren instrumentos especializados $(18,19)$.

Cuando falla la extracción digital, se puede emplear un anoscopio o un espéculo vaginal con el fin de visualizar el objeto, empleando una pinza de Foerster. En algunas ocasiones, la mucosa puede adherirse firmemente al objeto generando un vacío que impide que el objeto sea extraído. Se puede pasar una sonda Foley por detrás del objeto con insuflación de aire con el fin de quitar el vacío, a su vez con el paso de este y la insuflación del balón puede permitir la remoción con una tracción sutil (20).

La ventaja de la anestesia raquídea o general es la reducción del tono del esfínter anal que inhibe el espasmo, que mejora la visualización del objeto y aumenta sustancialmente la posibilidad de retiro por vía transanal (21).

En la literatura se han planteado diferentes técnicas de extracción de cuerpos extraños utilizando diferentes instrumentos dentro de los que se describen las asas de polipectomía, fórceps, sonda Foley, fórceps obstétricos, extractores de vacío, balones de dilatación, cilindros plásticos y espátulas vaginales.

En caso de que la extracción manual, extracción bajo anestesia, el TAMIS y la sigmoidoscopia flexible fallen, es necesaria la intervención quirúrgica. Se plantea como primera opción el abordaje laparoscópico. Con los instrumentos de cirugía mininamente invasiva se puede manipular transmuralmente y empujar el objeto distalmente. La cirugía abierta se reserva para casos de perforaciones rectales bajas, contaminación severa de la cavidad peritoneal y cuando no hay experiencia con esta técnica.

En el caso de laceraciones de la mucosa, edema o erosiones, se debe observar al paciente intrahospitalariamente (Figura 5).

\section{COMPLICACIONES}

Aunque se han planteado algunas a lo largo del artículo, aquí se enuncian las más importantes y relevantes:

- Perforación aguda, subaguda y crónica: fundamentalmente en caso de objetos punzantes. Las zonas de estrechez, angulación o fondos de saco del tracto digestivo y zonas de bridas son las que tienen mayor número de perforaciones, ocurriendo en un $75 \%$ de los casos en el área ileocecal (22).

- Absceso intramural, intrahepático, subfrénico o intraabdominal.

- Fístula intestinal, urinaria o enterocutánea.

- Migración del objeto a órganos vecinos (23).

- Obstrucción intestinal por impactación: las zonas anatómicas de impactación de los objetos extraños que causan obstrucción intestinal son la válvula ileocecal, ciego, apéndice y ano (24).

- Hemorragia digestiva por erosión de la pared del tubo digestivo.

- Intoxicación por absorción del material degradado.

- Sepsis.

- Síndrome de disfunción orgánica múltiple.

- Trastornos psicológicos en caso de agresiones sexuales.

- Muerte secundaria a sepsis y disfunción orgánica múltiple $(9,25)$.

\section{Fuente de financiación}

No hubo ningún apoyo financiero. 


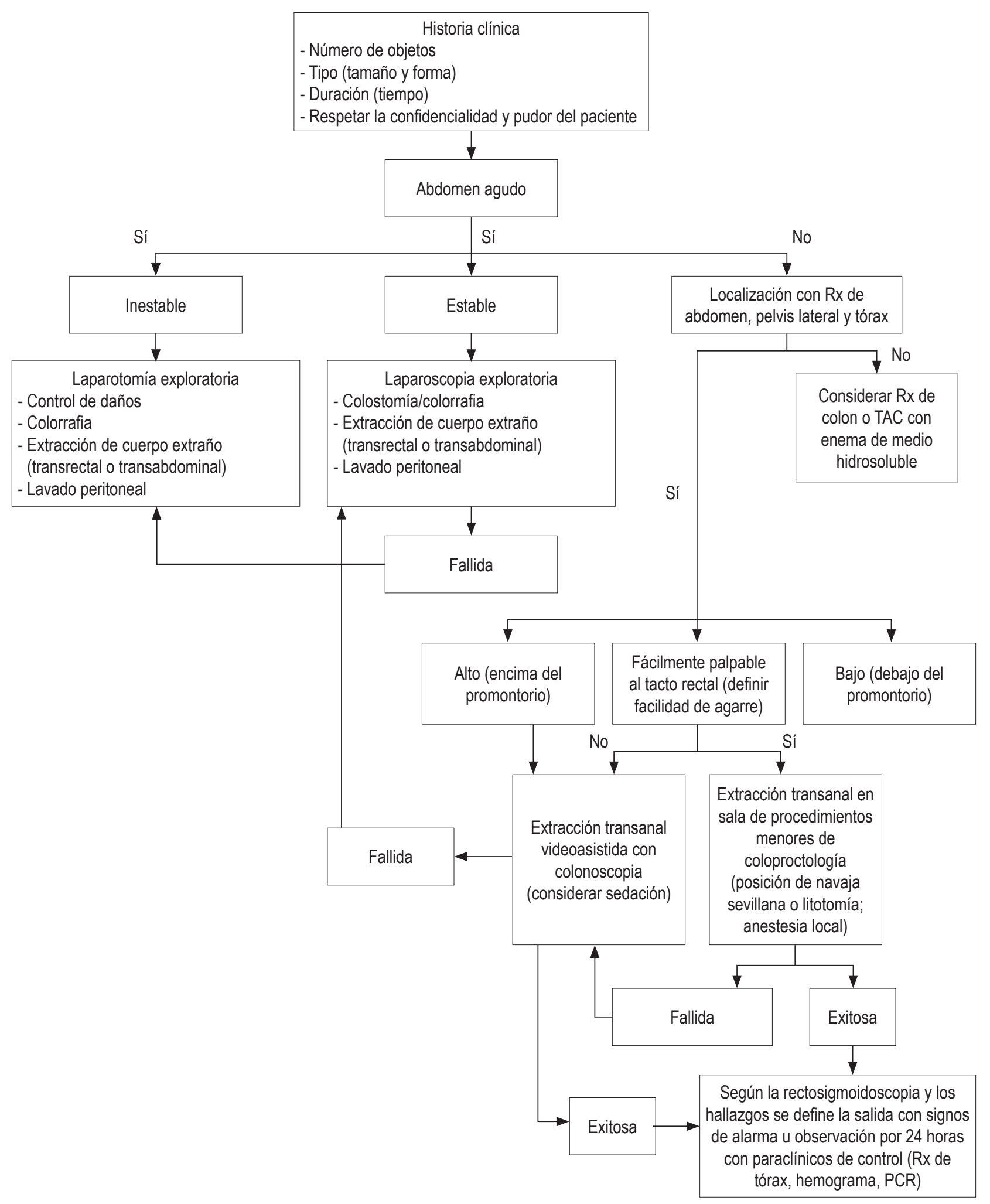

Figura 5. Algoritmo de manejo. 


\section{REFERENCIAS}

1. Ayantunde AA, Oke T. A review of gastrointestinal foreign bodies. Int J Clin Pract. 2006;60(6):735-9. https://doi. org/10.1111/j.1368-5031.2006.00709.x.

2. Desai B. Visual diagnosis: Rectal foreign body: A primer for emergency physicians. Int J Emerg Med. 2011;4:73. https://doi.org/10.1186/1865-1380-4-73.

3. Ozbilgin M, Arslan B, Yakut MC, et al. Five years with a rectal foreign body: A case report. Int J Surg Case Rep. 2015;6C:2103. https://doi.org/10.1016/j.ijscr.2014.11.053.

4. Clarke DL, Buccimazza I, Anderson FA, et al. Colorectal foreign bodies. Colorectal Dis. 2005;7(1):98-103. https:// doi.org/10.1111/j.1463-1318.2004.00699.x.

5. Stack LB, Munter DW. Foreign bodies in the gastrointestinal tract. Emerg Med Clin North Am. 1996;14(3):493-521. https://doi.org/10.1016/S0733-8627(05)70264-9.

6. Odagiri $\mathrm{H}$, Yasunaga $\mathrm{H}, \mathrm{Matsui} \mathrm{H}$, et al. Difference in outcomes of rectal foreign bodies between males and females: a retrospective analysis of a national inpatient database in Japan. Digestion. 2015;92(3):165-70. https://doi.org/10.1159/000439125.

7. Rodríguez-Hermosa JI, Codina-Cazador A, Sirvent JM, et al. Surgically treated perforations of the gastrointestinal tract caused by ingested foreign bodies. Colorectal Dis. 2008; 10(7):7017. https://doi.org/10.1111/j.1463-1318.2007.01401.x.

8. Smith MT, Wong RK. Foreign bodies. Gastrointest Endosc Clin N Am. 2007;17(2):361-82, vii. https://doi. org/10.1016/j.giec.2007.03.002.

9. Goldberg JE, Steele SR. Rectal foreign bodies. Surg Clin North Am. 2010;90(1):173-84. https://doi.org/10.1016/j. suc.2009.10.004.

10. Ayantunde AA. Approach to the diagnosis and management of retained rectal foreign bodies: clinical update. Tech Coloproctol. 2013;17(1):13-20. https://doi.org/10.1007/ s10151-012-0899-1.

11. Cohen JS, Sackier JM. Management of colorectal foreign bodies. J R Coll Surg Edinb. 1996;41(5):312-5.

12. Lake JP, Essani R, Petrone P, et al. Management of retained colorectal foreign bodies: predictors of operative intervention. Dis Colon Rectum. 2004;47(10):1694-8. https://doi. org/10.1007/s10350-004-0676-4.

13. Huang WC, Jiang JK, Wang HS, et al. Retained rectal foreign bodies. J Chin Med Assoc. 2003;66(10):607-12.
14. Cawich SO, Thomas DA, Mohammed F, et al. A management algorithm for retained rectal foreign bodies. Am J Mens Health. 2017;11(3):684-692. https://doi. org/10.1177/1557988316680929.

15. Fry RD. Anorectal trauma and foreign bodies. Surg Clin North Am. 1994;74(6):1491-505. https://doi. org/10.1016/S0039-6109(16)46494-7.

16. Tatar C, Karşıdağ T, Hut A. Successful endoscopic removal of a foreign body in the rectum. Turk J Gastroenterol. 2014;25(4):442-3. https://doi.org/10.5152/tjg.2014.5136.

17. Rodrigues FG, Campos JB, Silva GD, et al. Endoscopic ultrasound in the diagnosis of foreign bodies of the colon and rectum. Rev Assoc Med Bras (1992). 2016;62(9):818-21. https://doi.org/10.1590/1806-9282.62.09.818.

18. Bak Y, Merriam M, Neff M, et al. Novel approach to rectal foreign body extraction. JSLS. 2013;17(2):342-5. https:// doi.org/10.4293/108680813X13654754534233.

19. Cawich SO, Mohammed F, Spence R, et al. Colonic foreign body retrieval using a modified TAMIS technique with standard instruments and trocars. Case Rep Emerg Med. 2015;2015:815616. https://doi.org/10.1155/2015/815616.

20. Ayantunde AA, Unluer Z. Increasing trend in retained rectal foreign bodies. World J Gastrointest Surg. 2016;8(10):679-84. https://doi.org/10.4240/wjgs.v8.i10.679.

21. Cirocco WC. Anesthesia facilitates the extraction of rectal foreign bodies. Gastrointest Endosc. 2000;52(3):452-3. https://doi.org/10.1067/mge.2000.108045.

22. Flint LM, Vitale GC, Richardson JD, et al. The injured colon: relationships of management to complications. Ann Surg. 1981;193(5):619-23. https://doi.org/10.1097/00000658198105000-00012.

23. Chiu WK, Hsiao CW, Kang JC, et al. Intrapelvic migration with long-term retention of a rectal thermometer: a case report. Clin Pediatr (Phila). 2007;46(7):636-8. https://doi. org/10.1177/0009922807300701.

24. Hoare D, Akbar F, Maw A. Comment on 'Colorectal foreign bodies: a systematic review'. Colorectal Dis. 2011;13(1):108. https://doi.org/10.1111/j.1463-1318.2010.02503.x.

25. Kurer MA, Davey C, Khan S, et al. Colorectal foreign bodies: a systematic review. Colorectal Dis. 2010;12(9):851-61. https://doi.org/10.1111/j.1463-1318.2009.02109.x. 